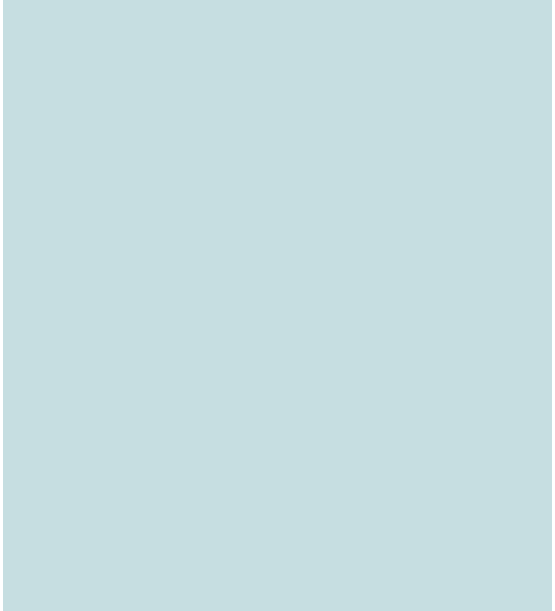

\title{
Multi-Ethnic Study of
} Atherosclerosis: Association

\section{between Left Atrial Function Using Tissue Tracking from Cine MR Imaging and Myocardial Fibrosis ${ }^{1}$}

Masamichi Imai, PhD, MD

Bharath Ambale Venkatesh, PhD

Sanaz Samiei, BSc

Sirisha Donekal, MD

Mohammadali Habibi, MD

Anderson C. Armstrong, MD

Susan R. Heckbert, MD, MPH, PhD

Colin 0. Wu, PhD

David A. Bluemke, PhD, MD

João A. C. Lima, MD
1 From the Division of Cardiology, Department of Medicine, Johns Hopkins University, $600 \mathrm{~N}$ Wolfe St, Blalock 524 Baltimore, MD 21287-0409 (M.I., B.A.V., S.S., S.D., A.C.A., J.A.C.L.); Department of Cardiology, Johns Hopkins Hospital, Baltimore, Md (M.H.); Cardiovascular Health Research Unit, Washington University, St Louis, Mo (S.R.H.); and Office of Biostatistics Research, National Heart, Lung and Blood Institute, National Institutes of Health, Bethesda, Md (C.O.W., D.A.B.). From the 2013 RSNA Annual Meeting. Received September 3, 2013; revision requested October 21; revision received February 27, 2014; accepted March 20; final version accepted May 14. Address correspondence to J.A.C.L. (e-mail: jlima1@jhmi.edu).
Purpose:

Materials and Methods:

Results:

Conclusion:

${ }^{\circ}$ RSNA, 2014

Online supplemental material is available for this article. replacement and diffuse myocardial fibrosis processes.

Clinical trial registration no. NCT00005487

To investigate the association between left atrial (LA) function and left ventricular myocardial fibrosis using cardiac magnetic resonance (MR) imaging in a multi-ethnic population.

For this HIPAA-compliant study, the institutional review board at each participating center approved the study protocol, and all participants provided informed consent. Of 2839 participants who had undergone cardiac MR in 2010-2012, 143 participants with myocardial scar determined with late gadolinium enhancement and 286 age, sex-, and ethnicity-matched control participants were identified. LA volume, strain, and strain rate were analyzed by using multimodality tissue tracking from cine MR imaging. T1 mapping was applied to assess diffuse myocardial fibrosis. The association between LA parameters and myocardial fibrosis was evaluated with the Student $t$ test and multivariable regression analysis.

The scar group had significantly higher minimum LA volume than the control group (mean, $22.0 \pm 10.5$ [standard deviation] vs $19.0 \pm 7.8, P=.002)$ and lower LA ejection fraction $(45.9 \pm 10.7$ vs $51.3 \pm 8.7, P<.001)$, maximal LA strain $\left(S_{\text {max }}\right)(25.4 \pm 10.7$ vs $30.6 \pm 10.6, P<.001)$ and maximum $\mathrm{LA}$ strain rate $\left(\mathrm{SR}_{\max }\right)(1.08 \pm 0.45$ vs 1.29 $\pm 0.51, P<.001)$, and lower absolute LA strain rate at early diastolic peak $\left(\mathrm{SR}_{\mathrm{E}}\right)(-0.77 \pm 0.42$ vs $-1.01 \pm 0.48$, $P<.001)$ and LA strain rate at atrial contraction peak $\left(\mathrm{SR}_{\mathrm{\alpha}}\right)(-1.50 \pm 0.62 \mathrm{vs}-1.78 \pm 0.69, P<.001)$ than the control group. T1 time 12 minutes after contrast material injection was significantly associated with $S_{\max }(\beta$ coefficient $=0.043, P=.013), \mathrm{SR}_{\max }(\beta$ coefficient $=0.0025, P$ $=.001), \mathrm{SR}_{\mathrm{E}}(\beta$ coefficient $=-0.0016, P=.027)$, and $\mathrm{SR}_{\mathrm{A}}$ ( $\beta$ coefficient $-0.0028, P=.01$ ) in the regression model. T1 time 25 minutes after contrast material injection was significantly associated with $\mathrm{SR}_{\max }(\beta$ coefficient $=0.0019$, $P=.016)$ and $\mathrm{SR}_{\mathrm{A}}(\beta$ coefficient $=-0.0022, P=.034)$.

Reduced LA regional and global function are related to both 
eft atrial (LA) size has shown association with cardiovascular risk and clinical outcomes (1-3). LA size and function reflect the effect of changes in left ventricular (LV) filling, structure, and function. Incremental LV preload caused by diastolic dysfunction affects LA architecture, influencing its performance, as well. In addition, myocardial damage, including myocardial infarction or other causes, frequently leads to impairment of LV filling consistent with the degree of $L V$ remodeling, and finally results in LA enlargement $(4,5)$ and impaired performance. In this regard, LA ejection fraction (EF) has been proposed as an independent predictor of clinical outcomes in a population of patients with myocardial infarction (6).

Cardiac magnetic resonance (MR) imaging is a useful noninvasive modality for diagnosing and monitoring the course of cardiac diseases. Late gadolinium enhancement (LGE) cardiac MR allows us to detect and quantify myocardial infarction and myocardial replacement scar $(7,8)$. Techniques such as T1 mapping that directly measure T1 relaxation times of the tissue are useful to assess diffuse interstitial myocardial fibrosis $(9,10)$. Myocardial T1 times have been correlated with myocardial fibrosis by using histologic synthesis and degradation assessment of collagen degeneration (11).

Clinically, LA function has been mainly assessed by using speckle

\section{Advance in Knowledge}

- Multimodality tissue tracking, a tool for tracking a cine image using a template-matching algorithm is introduced; left atrial (LA) parameters are associated with both regional replacement and diffuse myocardial fibrosis in the left ventricular (LV) wall; LA function may be more sensitive to changes occurring from myocardial fibrosis as compared with LA structure; increased LA volume and decreased LA function are associated with increased LV fibrosis. tracking echocardiography. LA strain and strain rate, measured by echocardiography, were suggested as independent and additional predictors of cardiovascular outcomes $(12,13)$. Cardiac MR is considered to be a useful modality for measuring LA volume $(14,15)$. LA dilatation has been studied by using cardiac MR in patients with ST-segment elevation myocardial infarction (6).

LA wall tissue tracking of cine cardiac MR images potentially provides more detailed information on timedependent changes in LA volume, strain, and strain rate. We hypothesized that LA function from cardiac MR would be a sensitive marker of LV myocardial fibrosis. The association of LA function with scar replacement and diffuse myocardial fibrosis remains unclear. The aim of this study was to investigate the association between LA function and LV myocardial fibrosis by using cardiac MR in a multiethnic population.

\section{Materials and Methods}

The Multi-Ethnic Study of Atherosclerosis (MESA) is an observational cohort study to investigate the prevalence, correlates, and progression of subclinical cardiovascular disease (CVD), initiated in 2000 across six centers in the United States (Baltimore, Md; Chicago, Ill; Forsyth County, North Carolina; Los Angeles County, California; Northern Manhattan, NY; and St Paul, Minn) (16). MESA participants were 45-84 years of age and were asymptomatic of clinical CVD in 2000-2002 at enrollment. Participants identified themselves as white (38\%), Chinese (11\%), African American (28\%), or Hispanic $(23 \%)$. In the follow-up examination in 2010-2012, a total of

\section{Implication for Patient Care}

- LA function can be a predictor of subclinical myocardial fibrosis; the measure of LA function can be clinically useful for follow-up for patients with high risk for myocardial infarction.
2839 participants underwent cardiac MR. All examinations and components of the Health Insurance Portability and Accountability Act-compliant MESA study were approved by the institutional review boards of all six centers, and informed consent for participation in this research was obtained from all participants.

\section{Population}

Among the 2839 MESA participants with cardiac MR in the follow-up study, 1661 (58.5\%) provided consent and underwent imaging with gadolinium enhancement. LGE revealed that 146 of the 1661 participants $(8.8 \%)$ had myocardial replacement scar. Three of

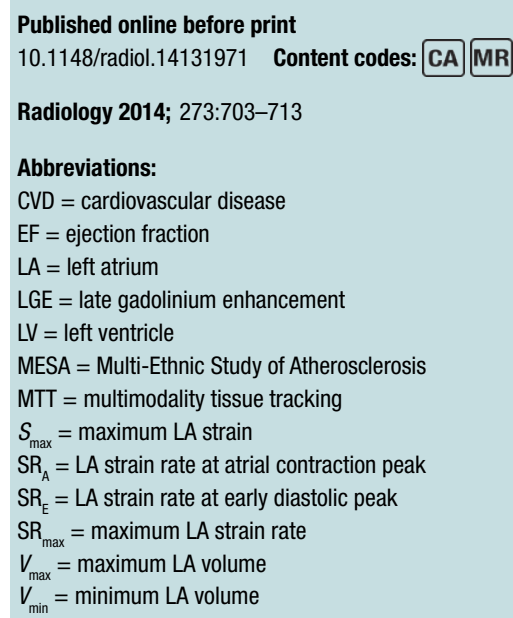

\section{Author contributions:}

Guarantors of integrity of entire study, M.I., B.A.V., C.O.W., J.A.C.L.; study concepts/study design or data acquisition or data analysis/interpretation, all authors; manuscript drafting or manuscript revision for important intellectual content, all authors; approval of final version of submitted manuscript, all authors; literature research, M.I., M.H., A.C.A., J.A.C.L.; clinical studies, M.I., S.D., D.A.B.; experimental studies, M.I.; statistical analysis, M.I., B.A.V., S.D., A.C.A., C.0.W.; and manuscript editing, M.I., B.A.V., S.S., S.D., M.H., A.C.A., J.A.C.L.

\section{Funding:}

This research was supported by the National Heart, Lung, and Blood Institute (grants N01-HC-95159-N01-HC-95166, N01-HC-95168) and by the National Center for Research Resources (grants UL1-RR-024156 and UL1-RR-025005), National Institutes of Health. C.O.W. and D.A.B. are employees of the National Institutes of Health.

Clinical trial registration no. NCT00005487

Conflicts of interest are listed at the end of this article. 
146 participants were excluded from the LA analysis because of cardiac MR technical limitations. Thus, 143 participants were ultimately included in the myocardial scar group (hereafter, scar group) of the LA analysis (Fig E1 [online]). From 1661 participants with gadolinium-enhanced images, we identified 286 participants without scar as control participants; the control group was matched for age, sex, and ethnicity with the scar group. Information was collected on traditional CVD risk factors as part of the follow-up examination, including body mass index, systolic blood pressure, heart rate, use of antihypertensive medication, glucose level abnormality (normal, impaired fasting glucose level, or diabetes mellitus), serum lipid levels, and smoking status. Of all 429 participants from both groups, 312 participants were also examined for interstitial fibrosis with T1 mapping.

\section{MR Imaging Protocol}

All participants underwent cardiac MR by using a 1.5-T whole-body MR imaging unit (Avanto; Siemens Medical Systems, Erlangen, Germany). The cine images of 12 short-axis sections, one two-chamber view, and one four-chamber view were obtained by using a steady-state free precession sequence with the following imaging parameters: repetition time msec/echo time msec, approximately 3.8/1.6 (minimized); matrix size, 256 $\times 205$; field of view, $360 \times 360 \mathrm{~mm}$; spatial resolution, $1.4 \times 1.7 \mathrm{~mm}$; section thickness, $8 \mathrm{~mm}$; number of phases, 40; and generalized autocalibrating partially parallel acquisition factor, two. Two- and four-chamber cine images were used for LA analysis. The short- and long-axis cine images were used to measure LV enddiastolic volume and myocardial volume by using numerical integration of the model surfaces using guide-point modeling (CIM version 4.6, Auckland MR Imaging Research Group, University of Auckland, Auckland, New Zealand). LV mass was calculated from myocardial volume, assuming a myocardial density of $1.05 \mathrm{~g} / \mathrm{mL}$. LV mass-to-volume ratio was obtained as LV mass divided by $L V$ end-diastolic volume (17). LGE MR images were obtained 15 minutes after an intravenous bolus injection of gadopentetate dimeglumine (Magnevist; Bayer HealthCare Pharmaceuticals, Montville, NJ), $0.15 \mathrm{mmol} / \mathrm{kg}$. T1 mapping was used to assess diffuse myocardial fibrosis. One short-axis image in the midventricular level was acquired by using modified look-locker inversion recovery (9) at three phases (nonenhanced, 12 minutes after contrast material injection, and 25 minutes after contrast material injection) by using the following imaging parameters: 2.2/1.1; flip angle, $35^{\circ}$; matrix size, $192 \times 183$; field of view, $360 \times 360$ $\mathrm{mm}$; section thickness, $8 \mathrm{~mm}$; and generalized autocalibrating partially parallel acquisition factor, two.

\section{LGE Analysis and T1 Mapping}

LGE was used to detect the presence of regional scar replacement. The images were analyzed with software (Qmass; Medis, Leiden University Medical Center, Leiden, the Netherlands) by a radiologist with more than 5 years of experience doing the same. The region of interest for myocardium was manually placed at a stack of 8-10 short-axis sections over the whole cardiac cycle. The scar replacement area was then detected as the area with increased signal intensity manually by the user for each section where enhancement was observed.

T1 mapping was performed by using research software (MASS; Medis, Leiden University Medical Center) to postprocess modified look-locker inversion-recovery source images at three phases (nonenhanced, $12 \mathrm{mi}$ nutes after contrast material injection, and 25 minutes after contrast material injection). LV endocardial and epicardial borders were semiautomatically traced with pixel-by-pixel fit using the Levenberg-Marquardt algorithm in each image over the whole cardiac cycle. The epicardial structure and blood pool were carefully excluded from the contours. This analysis was performed by one radiologist with more than 2 years of experience in analyzing T1 mapping images.

\section{LA Analysis by Using Tissue Tracking}

LA function was analyzed by using a tissue tracking method with semiautomated software (multimodality tissue tracking [MTT] version 5.0; Toshiba, Tochigi, Japan) by a trained radiologist (M.I.). LA endocardial and epicardial borders were manually drawn at a reference frame, the ventricular end-systolic frame just before the mitral valve opening (Fig 1). The software then propagated these borders across the cardiac cycle automatically by using a template-matching algorithm. The software recorded a characteristic pixel pattern of each $10 \times$ $10-\mathrm{mm}$ square area in the reference frame; an area with an identical pixel pattern was recognized in the next frame that maximized the similarity evaluated by cross-correlation between the square areas. This procedure was repeated for all pixels in each image and for each frame to track the borders throughout the whole cardiac cycle. LA volume was calculated by using the biplane modified Simpson method (18). LA strain was calculated by using an automated frame-to-frame pixel pattern-matching technique (19). LA strain and strain rate were calculated from the average of the global values in the longitudinal direction (perpendicular to the mitral valve plane) obtained from two- and four-chamber views. Maximum LA volume $\left(V_{\max }\right)$, minimum LA volume $\left(V_{\min }\right)$, maximum LA strain $\left(S_{\text {max }}\right)$, maximum LA strain rate $\left(\mathrm{SR}_{\mathrm{max}}\right)$, LA strain rate at early diastolic peak $\left(\mathrm{SR}_{\mathrm{E}}\right)$, and $\mathrm{LA}$ strain rate at atrial contraction peak $\left(\mathrm{SR}_{\mathrm{A}}\right)$ were obtained as relevant LA parameters for this study (Fig 2). All $V_{\text {max }}$ and $V_{\text {min }}$ were indexed according to body surface area in this study. LA EF was also calculated as follows: $\mathrm{LA} \mathrm{EF}=\left(V_{\max }-\right.$ $\left.V_{\min }\right) / V_{\max }$.

\section{Statistical Analysis}

Statistical analysis was performed with software (Stata, version 12; Stata Corp, College Station, Tex). LA function parameters and the participants' 

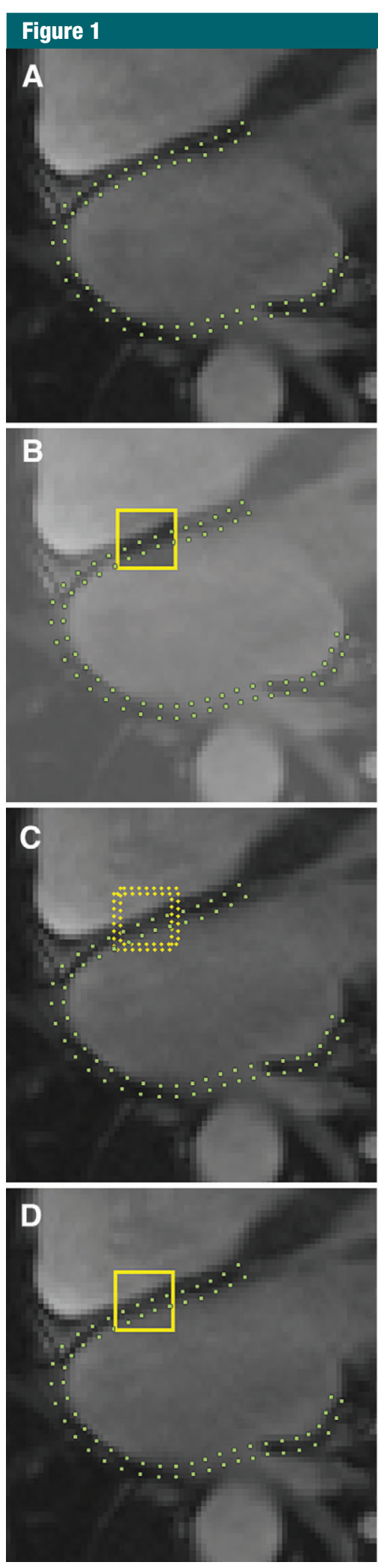
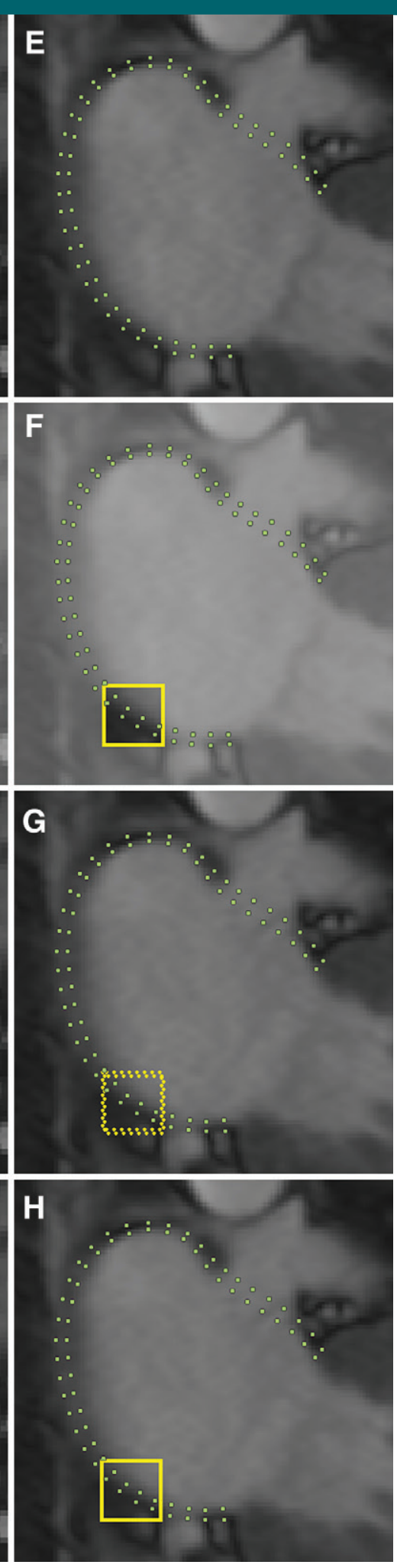

Figure 1: $\quad$ LA border was set on, $E$, two- and, $A$, four-chamber views. $B, F$, MTT read characteristic pixel pattern in each 10 $\times 10-\mathrm{mm}$ area (yellow square) as template piece of image. $C, G$, The identified area to the template image was searched in the next frame, and, $D, H$, then the best matching area was determined as the new location of the tracking. Repeating the algorithm, LA wall was tracked across a cardiac cycle. All images were obtained with the same parameters (3.8/1.6).

baseline data were evaluated by using the Student $t$ test for continuous variables and the $\chi^{2}$ test for categorical variables.

The association of LA function parameters with myocardial scar was assessed by using multivariable linear regression analysis. The regression models, with each LA parameter as a dependent variable, included presence of scar as an independent variable and demographic characteristics (age, sex, and ethnicity), traditional CVD risk factors, and LV remodeling as covariates. LV mass-to-volume ratio was used as the representative for $\mathrm{LV}$ remodeling (17). A difference with a $P$ value of less than .05 was considered to be significant.

The association of LA parameters with T1 time was assessed by using the Pearson correlation and multivariable linear regression analysis. The regression models for each LA parameter versus T1 time (nonenhanced, 12 minutes after contrast agent injection, or 25 minutes after contrast agent injection) included presence of scar, demographic characteristics, and weight. The adjustment for weight was added because the administered dose of gadolinium-based contrast agent was determined by the weight.

To evaluate the reproducibility of the measures of LA function with MTT, we assessed inter- and intraobserver variability. Twenty participants were randomly selected, and LA analysis was independently performed by two readers in a blinded fashion. The reproducibility was assessed with the intraclass correlation coefficient for each LA parameter. 

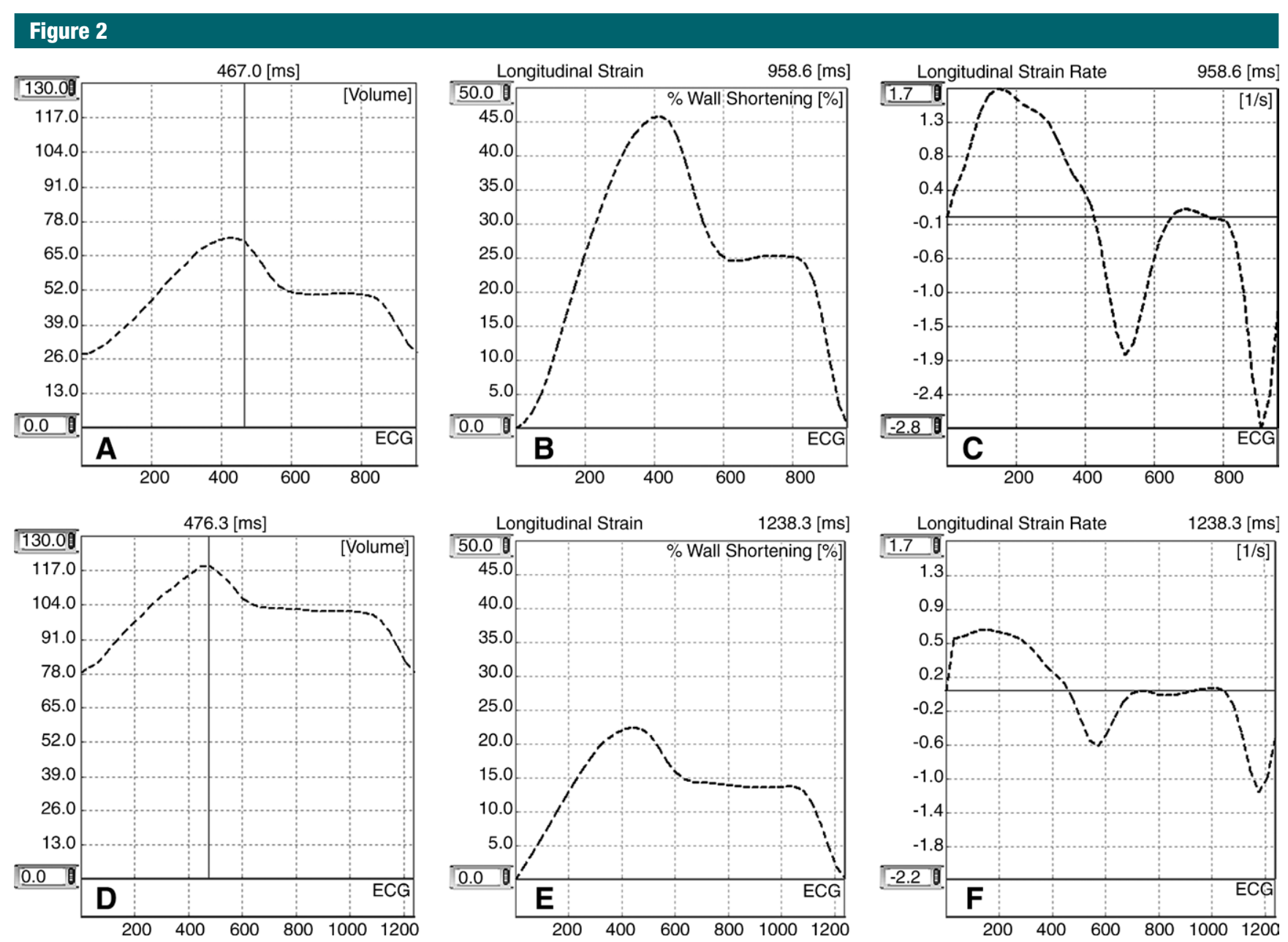

Figure 2: Representative plots of $L A$ volume, strain, and strain rate against time in, $A-C$, a control participant and, $D-F$, a participant with myocardial scar. Control participant: $A$, LA volume curve $\left(V_{\max }\right.$ and $V_{\min }$ were indexed), $B$, LA strain curve, and, $C$, LA strain rate curve. Participant with myocardial scar: $D$, LA volume curve, $E$, LA strain curve, and, F, LA strain rate curve. These graphs were obtained by using MTT (version 5.2; Toshiba) for comparison of participants who had myocardial scar with control participants, because scaling of $y$-axis is available in this version of MTT software, but it was not available in version 5.0 , which was actually used in this study. Both versions had the same capability for tissue tracking.

\section{Results}

\section{Participant Characteristics}

Table 1 shows the characteristics of this study population. Participants with scar had a mean age of 71.7 years, and $84.6 \%$ were men. A larger proportion of participants than in the overall population of participants in the follow-up study were white $(51.0 \%)$ and African American (30.1\%).

When we compared the scar group with the control group, matched for age, sex, and ethnicity, the proportion of participants using antihypertensive medications and the proportion of those who were current smokers were higher in the scar group.

\section{LA Parameters}

The mean LA parameters for the scar and control groups are shown in Table 2. The box plots of each LA parameter in the scar group and the control group are displayed in Figure E2 (online). Compared with the control group, the scar group had significantly higher $V_{\min }$, with a mean value of $22.0 \pm 10.5$ (standard deviation) versus $19.0 \pm 7.8$ for the control group $(P=.002)$; lower $\mathrm{LA} E F$, with a mean value of $45.9 \pm$
10.7 versus $51.3 \pm 8.7$ for the control group $(P<.001)$; lower $S_{\max }$, with a mean value of $25.4 \pm 10.7$ versus 30.6 \pm 10.6 for the control group $(P<.001)$; lower $\mathrm{SR}_{\max }$, with a mean value of 1.08 \pm 0.45 versus $1.29 \pm 0.51$ for the control group $(P<.001)$; higher $\mathrm{SR}_{\mathrm{E}}$, with a mean value of $-0.77 \pm 0.42$ versus $-1.01 \pm 0.48$ for the control group $(P$ $<.001$ ); and higher $\mathrm{SR}_{\wedge}$, with a mean value of $-1.50 \pm 0.62$ versus $-1.78 \pm$ 0.69 for the control group $(P<.001)$. For $V_{\max }$, the difference between the scar and control groups was not significant, with a mean value of $39.6 \pm$ 12.1 versus $38.3 \pm 10.8(P=.255)$. 
Table 3 displays the association of myocardial scar with LA parameters after adjustment for demographic characteristics, traditional CVD risk factors, and LV mass-to-volume ratio. These $\beta$ coefficients were $0.99\left(V_{\max }\right.$, $P=.315), 2.64\left(V_{\min }, P=.003\right),-4.93$
(LA EF, $P<.001),-4.29\left(S_{\max }, P<\right.$ $.001),-0.16\left(\mathrm{SR}_{\max }, P=.001\right), 0.19$ $\left(\mathrm{SR}_{\mathrm{E}}, P<.001\right)$, and $0.23\left(\mathrm{SR}_{\mathrm{A}}, P<\right.$ $.001)$ in the fully adjusted model. All the LA parameters that differed significantly between the scar group and the control group in unadjusted analyses

\section{Table 1}

\section{Participant Characteristics}

\begin{tabular}{|c|c|c|c|}
\hline Characteristics & Scar Group $(n=143)$ & Control Group $(n=286)$ & $P$ Value \\
\hline Age $(y)^{\star}$ & $71.7 \pm 9.3$ & $71.4 \pm 9.0$ & .727 \\
\hline Male sex ${ }^{\dagger}$ & $121(84.6)$ & 242 (84.6) & $>.99$ \\
\hline \multicolumn{4}{|l|}{ Ethnicity ${ }^{\dagger}$} \\
\hline White & $73(51.0)$ & $146(51.0)$ & $>.99$ \\
\hline Chinese & $5(3.5)$ & $10(3.5)$ & $>.99$ \\
\hline African American & $43(30.1)$ & $86(30.1)$ & $>.99$ \\
\hline Hispanic & $22(15.4)$ & $44(15.4)$ & $>.99$ \\
\hline Body mass index $\left(\mathrm{kg} / \mathrm{m}^{2}\right)^{\star}$ & $28.7 \pm 4.9$ & $27.9 \pm 4.2$ & .098 \\
\hline Systolic blood pressure $(\mathrm{mm} \mathrm{Hg})^{*}$ & $124.9 \pm 17.9$ & $122.8 \pm 18.3$ & .243 \\
\hline Heart rate (beats/min)* & $63.8 \pm 11.9$ & $63.2 \pm 10.2$ & .577 \\
\hline Antihypertensive medication use ${ }^{\dagger}$ & $90(62.9)$ & $137(47.9)$ & $.003^{\ddagger}$ \\
\hline Impaired fasting glucose level ${ }^{\dagger}$ & $40(28.0)$ & $68(23.8)$ & .112 \\
\hline Diabetes mellitus ${ }^{\dagger}$ & $31(21.7)$ & 38 (13.3) & $.01^{\ddagger}$ \\
\hline $\begin{array}{l}\text { High-density lipoprotein cholesterol } \\
\text { level }(\mathrm{mg} / \mathrm{dL})^{\star}\end{array}$ & $51.5 \pm 15.2$ & $50.8 \pm 15.4$ & 655 \\
\hline Total cholesterol level $(\mathrm{mg} / \mathrm{dL})^{*}$ & $172.0 \pm 37.2$ & $171.5 \pm 34.8$ & .905 \\
\hline \multicolumn{4}{|l|}{ Smoking status ${ }^{\dagger}$} \\
\hline Former smoker & $72(50.3)$ & $168(58.7)$ & .615 \\
\hline Current smoker & $22(15.4)$ & $17(5.9)$ & $.006^{\ddagger}$ \\
\hline
\end{tabular}

Note.-To convert high-density lipoprotein cholesterol and total cholesterol levels to Système International units in millimoles per liter, multiply by .0259 .

* Data are means \pm standard deviations, except where otherwise indicated.

${ }^{\dagger}$ Data are numbers of participants, with percentages in parentheses, except where otherwise indicated.

$\ddagger P<.05$.

(Table 2) remained significantly associated with myocardial scar in the fully adjusted analyses.

The Pearson correlation of LA parameters to each $\mathrm{T} 1$ time is shown in Table 4 . The scatter plots of each LA parameter versus $\mathrm{T} 1$ time are displayed in Figures E3 and E4 (online) (12 and 25 minutes after contrast agent injection). T1 time for the nonenhanced phase was not associated with any LA parameter (Fig E5 [online]). Lower T1 time 12 minutes after contrast agent injection was significantly associated with higher $V_{\min }(R=-0.140$, $P=.013)$, lower LA EF $(R=0.213$, $P<.001)$, lower $S_{\max }(R=0.218, P$ $<.001)$, lower $\mathrm{SR}_{\text {max }}(R=0.254, P<$ $.001)$, higher $\operatorname{SR}_{\mathrm{E}}(R=-0.205, P<$ $.001)$, and higher $\mathrm{SR}_{\mathrm{A}}(R=-0.223$, $P<.001)$. T1 time 25 minutes after contrast agent injection showed similar associations as T1 time 12 minutes after contrast agent injection and was also significantly related to higher $V_{\text {max }}$ $(R=-0.131, P=.021)$. Table 5 shows the associations after adjustment for presence of scar, demographic characteristics, and weight. For T1 time 12 minutes after contrast agent injection, the associations with LA strain and strain rate remained significant: The $\beta$ coefficients were $0.043\left(S_{\max }, P=.013\right)$, $0.0025\left(\mathrm{SR}_{\max }, P=.001\right),-0.0016$ $\left(\mathrm{SR}_{\mathrm{E}}, P=.027\right)$, and $-0.0028\left(\mathrm{SR}_{\mathrm{A}}, P\right.$ $=.01)$ in the fully adjusted model. $\mathrm{T} 1$ time 25 minutes after contrast agent injection kept significant association

\section{Table 2}

\section{Comparison of LA Parameters between Scar and Control Groups}

\begin{tabular}{lcccc} 
LA Parameter & All $(n=429)$ & Scar Group $(n=143)$ & Control Group $(n=286)$ & PValue for Scar vs Control Group \\
\hline$V_{\text {max }}\left(\mathrm{mL} / \mathrm{m}^{2}\right)$ & $38.7 \pm 11.3$ & $39.6 \pm 12.1$ & $38.3 \pm 10.8$ & .255 \\
$V_{\min }\left(\mathrm{mL} / \mathrm{m}^{2}\right)$ & $20.0 \pm 8.9$ & $22.0 \pm 10.5$ & $19.0 \pm 7.8$ & $.002^{\star}$ \\
$\mathrm{LAEF}(\%)$ & $49.5 \pm 9.7$ & $45.9 \pm 10.7$ & $51.3 \pm 8.7$ & $<.001^{*}$ \\
$S_{\max }(\%)$ & $28.7 \pm 10.9$ & $25.4 \pm 10.7$ & $30.6 \pm 10.6$ & $<.001^{*}$ \\
$\mathrm{SR}_{\max }^{\dagger}$ & $1.22 \pm 0.50$ & $1.08 \pm 0.45$ & $1.29 \pm 0.51$ & 0.069 \\
$\mathrm{SR}_{\mathrm{E}}^{\dagger}$ & $-0.93 \pm 0.47$ & $-0.77 \pm 0.42$ & $-1.01 \pm 0.48$ & 0.051 \\
$\mathrm{SR}_{\mathrm{A}}^{\dagger}$ & $-1.69 \pm 0.67$ & $-1.50 \pm 0.62$ & $-1.78 \pm 0.69$ & 0.038 \\
\hline
\end{tabular}

Note.-Data are means \pm standard deviations. Effect size refers to the proportion of variance for each LA parameter by total variance. $V_{\max }$ and $V_{\min }$ were indexed to body surface area.

* $P<.05$.

${ }^{\dagger}$ Data are percentages per millisecond. 


\section{Table 3}

\section{Adjusted Associations of LA Parameters and Presence of Myocardial Scar}

\begin{tabular}{|c|c|c|c|c|c|c|c|c|c|}
\hline \multirow[b]{2}{*}{ LA Parameter } & \multicolumn{3}{|c|}{ Model 1} & \multicolumn{3}{|c|}{ Model 2} & \multicolumn{3}{|c|}{ Model 3} \\
\hline & $\beta$ Coefficient & $P$ Value & Effect Size & $\beta$ Coefficient & $P$ Value & Effect Size & $\beta$ Coefficient & $P$ Value & Effect Size \\
\hline$V_{\max }\left(\mathrm{mL} / \mathrm{m}^{2}\right)$ & 1.22 & .274 & 0.003 & 1.25 & .271 & 0.003 & 0.99 & .315 & 0.002 \\
\hline$V_{\min }\left(\mathrm{mL} / \mathrm{m}^{2}\right)$ & 2.90 & $.002^{\star}$ & 0.024 & 2.77 & $.003^{\star}$ & 0.020 & 2.64 & $.003^{\star}$ & 0.018 \\
\hline LA EF (\%) & -5.30 & $<.001^{\star}$ & 0.067 & -4.91 & $<.001^{\star}$ & 0.053 & -4.93 & $<.001^{\star}$ & 0.053 \\
\hline$S_{\max }(\%)$ & -5.14 & $<.001^{\star}$ & 0.049 & -4.20 & $<.001^{\star}$ & 0.031 & -4.29 & $<.001^{\star}$ & 0.032 \\
\hline $\mathrm{SR}_{\max }^{\dagger}$ & -0.20 & $<.001^{\star}$ & 0.037 & -0.16 & $.001^{\star}$ & 0.021 & -0.16 & $.001^{\star}$ & 0.021 \\
\hline $\mathrm{SR}_{\mathrm{E}}^{\dagger}$ & 0.23 & $<.001^{\star}$ & 0.051 & 0.18 & $<.001^{\star}$ & 0.031 & 0.19 & $<.001^{\star}$ & 0.032 \\
\hline $\mathrm{SR}_{\mathrm{A}}^{\dagger}$ & 0.27 & $<.001^{\star}$ & 0.036 & 0.23 & $<.001^{\star}$ & 0.025 & 0.23 & $<.001^{\star}$ & 0.024 \\
\hline
\end{tabular}

Note.-The $\beta$ coefficient expresses the mean difference in each LA parameter in the scar group versus the control group, after adjustment for the covariates in each model. Effect size refers to the proportion of variance for each LA parameter by total variance. The absolute values of $S_{E}$ and $S R_{A}$ are lower with the scar group even if their $\beta$ coefficient was positive, because the $S_{E}$ and $S_{A}$ were generally negative. A negative $\beta$ coefficient refers to a lower value (LA EF, $S_{\text {max }}$, and $\left.\mathrm{SR}_{\max }\right)$, and a positive $\beta$ coefficient refers to a higher value $\left(V_{\max }\right.$ and $\left.V_{\text {min }}\right)$. This is vice versa when the quantity being measured is itself negative (as seen in $\mathrm{SR}_{\mathrm{E}}$ and $\mathrm{SR}_{\mathrm{A}}$ ). Model 1 was adjusted for demographic data. Model 2 was adjusted for demographic data and traditional CVD risk factors. Model 3 was adjusted for demographic data, traditional CVD risk factors, and LV mass-to-volume ratio. Demographic data included age, sex, and ethnicity. Traditional CVD risk factors included body mass index, systolic blood pressure, heart rate, use of antihypertensive medication, plasma glucose status (impaired fasting glucose level or diabetes mellitus), serum lipid level status, and smoking status. $V_{\max }$ and $V_{\min }$ were indexed to body surface area.

* $P<.05$.

${ }^{\dagger}$ Data are percentages per millisecond.

\section{Table 4}

\section{Pearson Correlation between LA Parameters and T1 Values}

\begin{tabular}{lccc} 
& & \multicolumn{2}{c}{ T1 after Contrast Agent Injection } \\
\cline { 3 - 4 } LA Parameter & Nonenhanced T1 & 12 Minutes & 25 Minutes \\
\hline$V_{\max }$ & $-0.045(.424)$ & $-0.078(.172)$ & $-0.131(.021)^{\star}$ \\
$V_{\min }$ & $0.009(.87)$ & $-0.140(.013)^{\star}$ & $-0.180(.001)^{\star}$ \\
$\mathrm{LAEF}$ & $-0.080(.158)$ & $0.213(<.001)^{\star}$ & $0.202(<.001)^{\star}$ \\
$S_{\max }$ & $-0.053(.349)$ & $0.218(<.001)^{\star}$ & $0.198(<.001)^{\star}$ \\
$\mathrm{SR}_{\max }$ & $-0.032(.577)$ & $0.254(<.001)^{\star}$ & $0.232(<.001)^{\star}$ \\
$\mathrm{SR}_{\mathrm{E}}$ & $0.084(.138)$ & $-0.205(<.001)^{\star}$ & $-0.165(.004)^{\star}$ \\
$\mathrm{SR}_{\mathrm{A}}$ & $0.028(.624)$ & $-0.223(<.001)^{\star}$ & $-0.214(.001)^{\star}$ \\
\hline
\end{tabular}

Note.-Data are correlation coefficients, and numbers in parentheses are $P$ values. $V_{\max }$ and $V_{\min }$ were indexed to body surface area.

${ }^{*} P<.05$.

with LA strain rate: The $\beta$ coefficient values were $0.0019\left(\mathrm{SR}_{\max }, P=.016\right)$, and $-0.0022\left(\mathrm{SR}_{\mathrm{A}}, P=.034\right)$.

\section{Reproducibility of LA Analysis by Using MTT}

The intraobserver intraclass correlation coefficient was between 0.834 and 0.959 , and the interobserver intraclass correlation coefficient was between 0.779 and 0.984 for all measured LA parameters by using MTT (Table E1 [online]). Bland-Altman plots for intraobserver and interobserver reproducibility, respectively, are shown in Figures 3 and 4.

\section{Discussion}

To our knowledge, the finding of the association between multiple LA parameters and myocardial fibrosis has not previously been well established in the literature. The main findings of this study can be summarized as follows: (a) MTT can be used to measure multiple LA parameters with excellent intraobserver and interobserver reproducibility; (b) presence of myocardial scar, detected with LGE $(20,21)$, was associated with higher $V_{\text {min }}$, lower LA EF, lower maximum LA strain, and lower maximum absolute LA strain rate peaks but was not significantly associated with $V_{\max }$; (c) lower T1 time after contrast agent injection, reflecting greater diffuse myocardial fibrosis (22), was associated with higher LA volume, lower LA EF, lower LA strain, and lower LA strain rate.

Echocardiography is commonly used to measure LA size and function, and previous reports indicate that LA volume and strain measured by using echocardiography are important prognostic outcome predictors in patients who had myocardial infarction $(2,23)$. However, Doppler and speckle tracking echocardiography can be challenging for the measurement of LA function because of inconsistent image quality. Cardiac MR has been established with great accuracy and reproducibility (15), and then cardiac MR is considered a standard clinical technique to measure LA dimensions and volumes (24).

The most common method for strain analysis by using cardiac MR is tagged MR imaging (25). By using MR imaging tagging, more efficient methods have been proposed previously for measurement of LV strain (26). However, it 
is challenging to apply tagging methods to the measurement of LA strain. The LA wall is thinner than the $\mathrm{LV}$ or right ventricular walls, and frequently, it is too thin to allow adequate performance of tagged MR imaging.

We analyzed LA function with MTT by using a tissue-tracking method (27). This method was developed for the purpose of measuring wall strain in a less time-intensive manner. Strain is obtained by tracking cardiac chamber walls across the cardiac cycle efficiently. In tissue tracking, we can leverage the cine image included in a regular cardiac MR examination; an additional image required for the measurement of strain is not needed, as it is for tagged MR imaging. In addition, tissue tracking has no limitation in the assessment of diastolic relaxation when compared with tagged MR imaging, in which tags fade during the late diastolic phase because of $\mathrm{T} 1$ relaxation, which impairs the assessment of myocardial deformation during the atrial contraction and relaxation periods. The measurement of $\mathrm{LV}$ and right ventricular strain by using cine cardiac MR has been reported in a few previous studies $(28,29)$. It has been suggested that the measurement of LV strain by using tissue tracking with cine cardiac MR imaging was as useful as tagged MR imaging and speckle tracking echocardiography $(28,30,31)$. The interobserver reproducibility for tissue tracking was similar to echocardiography in the measurement of longitudinal LV global strain (28). In our study, we obtained good inter- and intraobserver agreement for LA strain analysis with this method of tissue tracking.

In the measurement of LA volume by using cardiac MR, Maceira et al (24) reported both straight and indexed mean $V_{\max }$ of $40 \mathrm{~mL} / \mathrm{m}^{2} \pm 6.7$ in 120 healthy subjects. In our study, we obtained a mean $V_{\text {max }}$ as $38.7 \mathrm{~mL} / \mathrm{m}^{2} \pm$ 11.3 for the entire group of MESA participants. Our results thus identified values similar to those in the previous report. $V_{\text {max }}$ for the scar group was not significantly different from that found in the control group. In contrast, $V_{\min }$ significantly increased in the scar group. This suggests that $V_{\min }$ is more

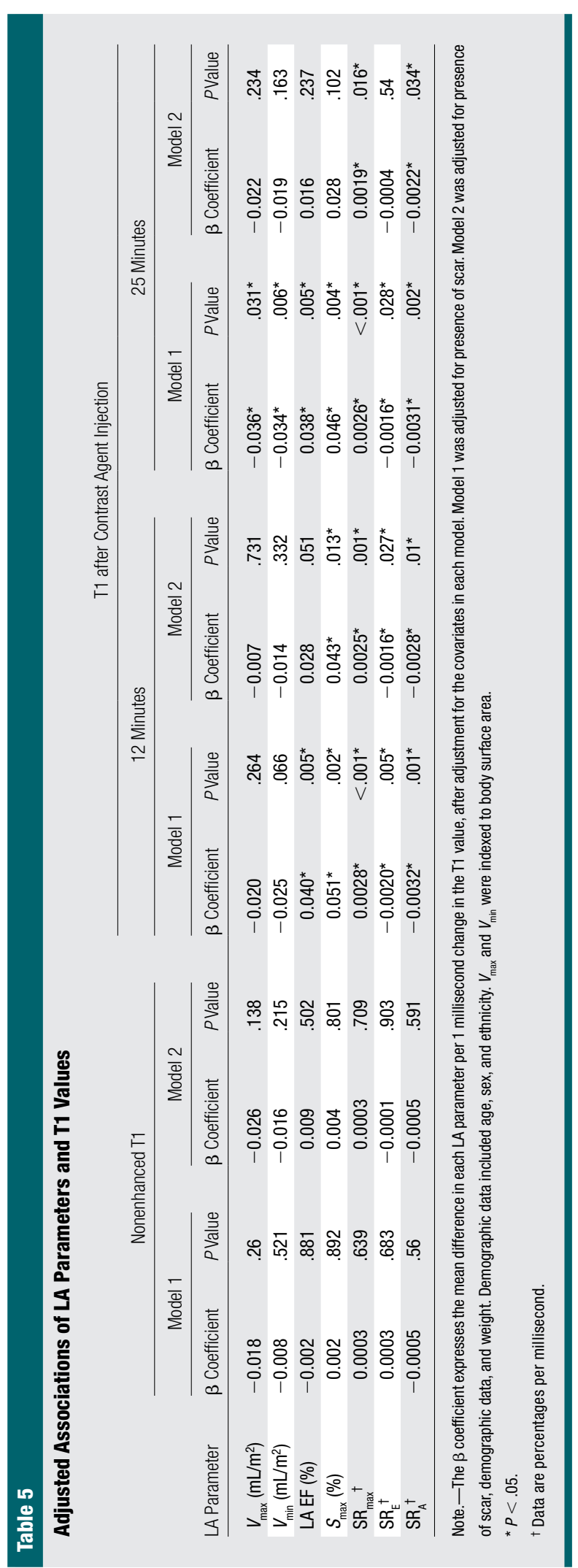




\section{Figure 3}
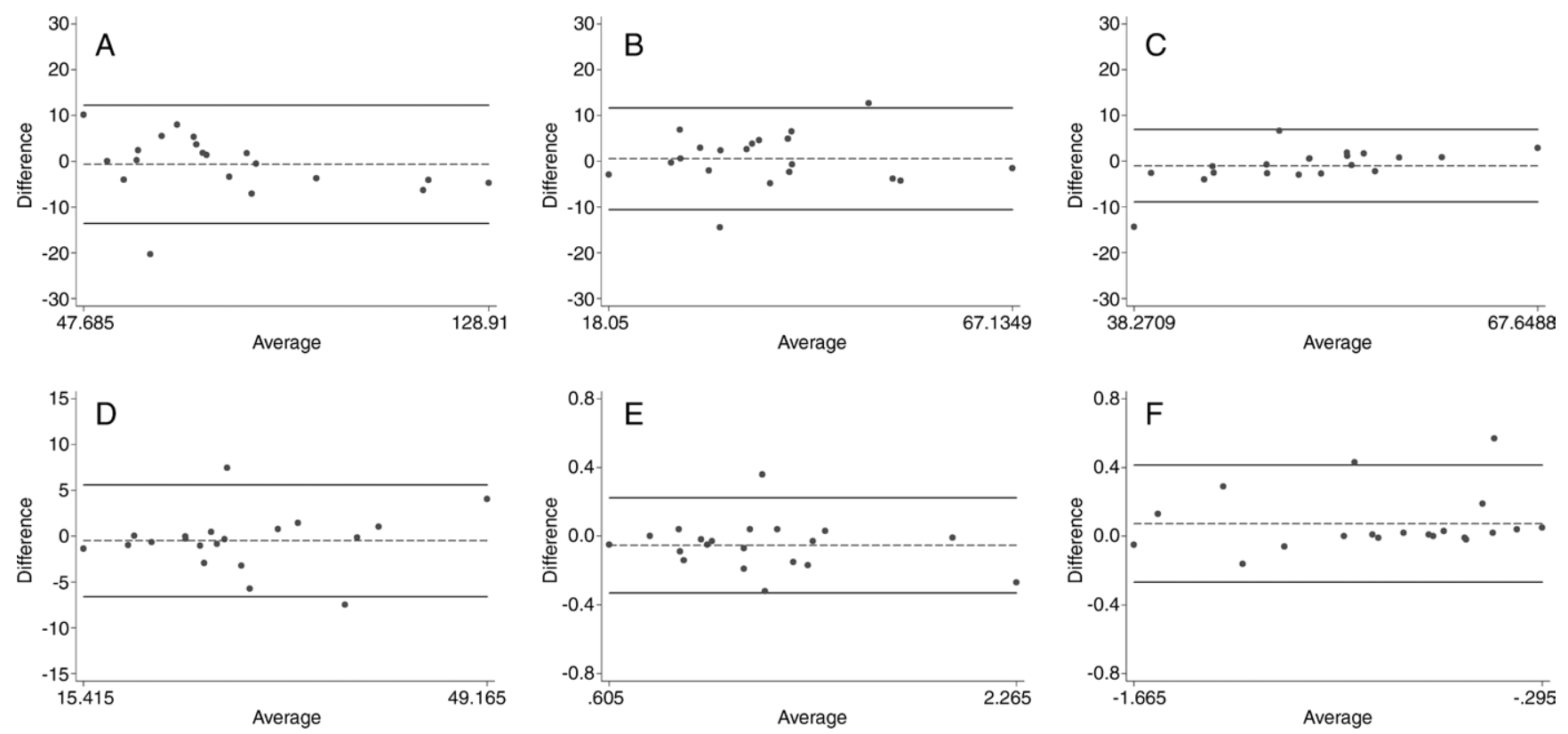

Figure 3: Bland-Altman plots for intraobserver reproducibility. $A, V_{\max ^{\prime}} B, V_{\min }, C, \mathrm{LA} E F, D, S_{\max }, E, \mathrm{SR}_{\max }$, $F, \mathrm{SR}_{\mathrm{E}}$, and $\mathrm{G}, \mathrm{SR}_{\mathrm{A}}$.

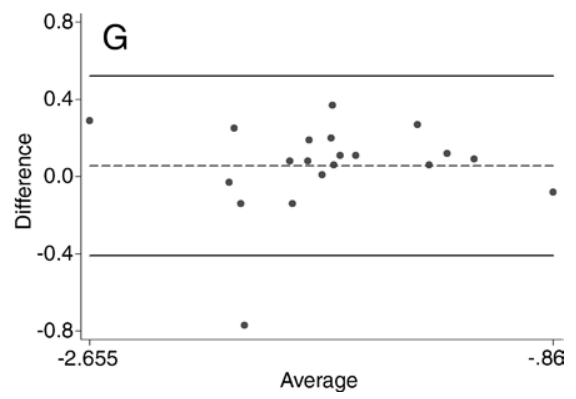

sensitively associated with myocardial scar. LA EF was also significantly decreased; that can be explained by the increase in $V_{\min }$ and similarity in $V_{\max }$. Although increased $V_{\max }$ is an important prognostic predictor in various cardiac diseases $(1-3,12,32,33)$, evaluating $V_{\min }$ and LA EF might aid in detecting early, subclinical LA filling impairment.

We demonstrated that LA strain and strain rate were significantly different between the subclinical myocardial scar group and the control group. All of the LA strain and strain rates were significant even after adjustment for demographic data, traditional risk factors, and LV remodeling level. This result suggests that LA strain analysis is of greater utility for detecting changes in LA function as a result of myocardial scar in the early stage before cardiac symptoms appear, in comparison with indexed LA volume.

More important, LA parameters were also associated with $\mathrm{T} 1$ times after contrast agent injection. To control for the effect of myocardial scar on such measurements, the first model was adjusted for presence of scar in the multiple regression analysis. In our study, reduced LA strain and strain rate were significantly associated with lower T1 time after contrast agent injection even after adjustment for all covariates, but LA volume was not associated with interstitial fibrosis indexed according to T1 times. This suggests that diffuse myocardial fibrosis is associated with reduced LA function specifically before significant changes in LA size can be observed, while presence of replacement scar in the left ventricle is associated with both LA size and function.

In conclusion, we have shown that reduced LA regional and global function are related to both replacement and diffuse myocardial fibrosis processes. LA regional and global function is also more sensitive to changes occurring from subclinical myocardial dysfunction, as compared with LA structure. MTT is a useful and reliable tool for LA structure and function assessment.

Our study had some limitations. In a few cases, the LA was difficult to measure because of extreme curvature, probably secondary to undue angulation of the imaging plane. If the LA was too severely curved, it appeared to be "collapsed," especially in the enddiastolic frame of either the two- or 


\section{Figure 4}
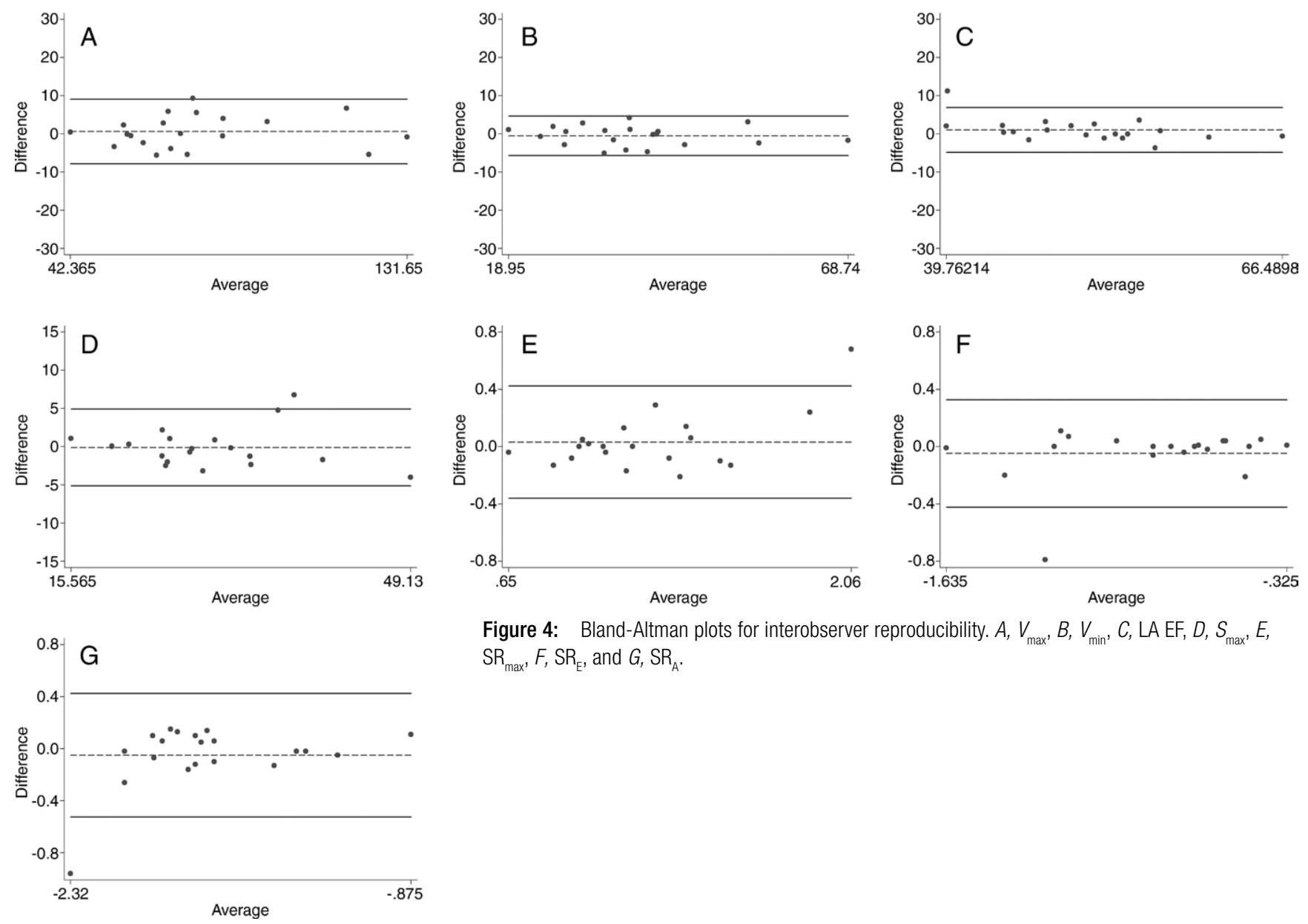

Figure 4: Bland-Altman plots for interobserver reproducibility. $A, V_{\max }, B, V_{\min }, C, L A E F, D, S_{\max }, E$, $\mathrm{SR}_{\text {max }}, F, \mathrm{SR}_{\mathrm{E}}$, and $G, \mathrm{SR}_{\mathrm{A}}$.

the four-chamber views, or both. MTT failed to track the LA wall in such cases. In this study, three participants were excluded from the scar group because they had an LA that was severely curved.

Acknowledgments: We thank the other investigators, the staff, and the participants of the MESA study for their valuable contributions. A full list of participating MESA investigators and institutions can be found at www.mesa-nhlbi.org.

Disclosures of Conflicts of Interest: M.I. disclosed no relevant relationships. B.A.V. disclosed no relevant relationships. S.S. disclosed no relevant relationships. S.D. disclosed no relevant relationships. M.H. disclosed no relevant relationships. A.C.A. disclosed no relevant relationships. S.R.H. disclosed no relevant relationships. C.O.W. disclosed no relevant relationships. D.A.B. disclosed no relevant relationships. J.A.C.L. disclosed no relevant relationships.

\section{References}

1. Møller JE, Hillis GS, Oh JK, et al. Left atrial volume: a powerful predictor of survival after acute myocardial infarction. Circulation 2003;107(17):2207-2212.

2. Benjamin EJ, D'Agostino RB, Belanger AJ, Wolf PA, Levy D. Left atrial size and the risk of stroke and death. The Framingham Heart Study. Circulation 1995;92(4):835-841.

3. Ammash NM, Seward JB, Bailey KR, Edwards WD, Tajik AJ. Clinical profile and outcome of idiopathic restrictive cardiomyopathy. Circulation 2000;101(21):24902496.

4. Lam W, Leano R, Haluska B, Marwick TH. Effects of myocardial ischaemia on left ventricular untwist and filling pressure. Heart 2011;97(9):757-761.

5. Abhayaratna WP, Seward JB, Appleton CP, et al. Left atrial size: physiologic determi- nants and clinical applications. J Am Coll Cardiol 2006;47(12):2357-2363.

6. Lonborg JT, Engstrom T, Moller JE, et al. Left atrial volume and function in patients following ST elevation myocardial infarction and the association with clinical outcome: a cardiovascular magnetic resonance study. Eur Heart $\mathrm{J}$ Cardiovasc Imaging 2013;14(2):118-127.

7. Ingkanisorn WP, Rhoads KL, Aletras AH, Kellman P, Arai AE. Gadolinium delayed enhancement cardiovascular magnetic resonance correlates with clinical measures of myocardial infarction. J Am Coll Cardiol 2004;43(12):2253-2259.

8. Cummings KW, Bhalla S, Javidan-Nejad C, Bierhals AJ, Gutierrez FR, Woodard PK. A pattern-based approach to assessment of delayed enhancement in nonischemic cardiomyopathy at MR imaging. RadioGraphics 2009;29(1):89-103. 
9. Messroghli DR, Radjenovic A, Kozerke S, Higgins DM, Sivananthan MU, Ridgway JP. Modified Look-Locker inversion recovery (MOLLI) for high-resolution T1 mapping of the heart. Magn Reson Med 2004;52(1):141-146.

10. Nles L, Pfluger H, Phrommintikul A, et al. Evaluation of diffuse myocardial fibrosis in heart failure with cardiac magnetic resonance contrast-enhanced T1 mapping. J Am Coll Cardiol 2008;52(19):1574-1580.

11. Flett AS, Hayward MP, Ashworth MT, et al. Equilibrium contrast cardiovascular magnetic resonance for the measurement of diffuse myocardial fibrosis: preliminary validation in humans. Circulation 2010;122(2): 138-144.

12. Rossi A, Temporelli PL, Quintana M, et al. Independent relationship of left atrial size and mortality in patients with heart failure: an individual patient meta-analysis of longitudinal data (MeRGE Heart Failure). Eur J Heart Fail 2009;11(10):929-936.

13. Cameli M, Lisi M, Focardi M, et al. Left atrial deformation analysis by speckle tracking echocardiography for prediction of cardiovascular outcomes. Am J Cardiol 2012;110(2): 264-269.

14. Järvinen V, Kupari M, Hekali P, Poutanen V-P. Assessment of left atrial volumes and phasic function using cine magnetic resonance imaging in normal subjects. Am J Cardiol 1994;73(15):1135-1138.

15. Grothues F, Smith GC, Moon JCC, et al. Comparison of interstudy reproducibility of cardiovascular magnetic resonance with two-dimensional echocardiography in normal subjects and in patients with heart failure or left ventricular hypertrophy. Am J Cardiol 2002;90(1):29-34.

16. Bild DE, Bluemke DA, Burke GL, et al. Multi-ethnic study of atherosclerosis: objectives and design. Am $\mathrm{J}$ Epidemiol 2002;156(9):871-881 .

17. Rodriguez CJ, Diez-Roux AV, Moran A, et al. Left ventricular mass and ventricular remodeling among Hispanic subgroups compared with non-Hispanic blacks and whites: MESA (Multi-ethnic Study of Atherosclerosis). J Am Coll Cardiol 2010;55(3): 234-242.

18. Ujino K, Barnes ME, Cha SS, et al. Twodimensional echocardiographic methods for assessment of left atrial volume. Am J Cardiol 2006;98(9):1185-1188.

19. Ogawa K, Hozumi T, Sugioka K, et al. Usefulness of automated quantitation of regional left ventricular wall motion by a novel method of two-dimensional echocardiographic tracking. Am J Cardiol 2006;98(11):1531-1537.

20. Kim RJ, Fieno DS, Parrish TB, et al. Relationship of MRI delayed contrast enhancement to irreversible injury, infarct age, and contractile function. Circulation 1999;100(19): 1992-2002.

21. Fieno DS, Kim RJ, Chen EL, Lomasney JW, Klocke FJ, Judd RM. Contrast-enhanced magnetic resonance imaging of myocardium at risk: distinction between reversible and irreversible injury throughout infarct healing. $J$ Am Coll Cardiol 2000;36(6):1985-1991.

22. Ng AC, Auger D, Delgado V, et al. Association between diffuse myocardial fibrosis by cardiac magnetic resonance contrast-enhanced $\mathrm{T}_{1}$ mapping and subclinical myocardial dysfunction in diabetic patients: a pilot study. Circ Cardiovasc Imaging 2012;5(1):51-59.

23. Antoni ML, ten Brinke EA, Atary JZ, et al. Left atrial strain is related to adverse events in patients after acute myocardial infarction treated with primary percutaneous coronary intervention. Heart 2011;97(16):1332-1337.

24. Maceira AM, Cosín-Sales J, Roughton M, Prasad SK, Pennell DJ. Reference left atrial dimensions and volumes by steady state free precession cardiovascular magnetic resonance. J Cardiovasc Magn Reson 2010;12:65

25. Zerhouni EA, Parish DM, Rogers WJ, Yang A, Shapiro EP. Human heart: tagging with MR imaging - a method for noninvasive as- sessment of myocardial motion. Radiology 1988;169(1):59-63.

26. Axel L, Montillo A, Kim D. Tagged magnetic resonance imaging of the heart: a survey. Med Image Anal 2005;9(4):376-393.

27. Helle-Valle TM, Yu WC, Fernandes VR, Rosen BD, Lima JA. Usefulness of radial strain mapping by multidetector computer tomography to quantify regional myocardial infarction. Am J Cardiol 2010;106(4):483491.

28. Kempny A, Fernández-Jiménez R, Orwat $\mathrm{S}$, et al. Quantification of biventricular myocardial function using cardiac magnetic resonance feature tracking, endocardial border delineation and echocardiographic speckle tracking in patients with repaired tetralogy of Fallot and healthy controls. J Cardiovasc Magn Reson 2012;14:32.

29. Schuster A, Morton G, Hussain ST, et al. The intra-observer reproducibility of cardiovascular magnetic resonance myocardial feature tracking strain assessment is independent of field strength. Eur J Radiol 2013;82(2):296-301.

30. Harrild DM, Han Y, Geva T, Zhou J, Marcus E, Powell AJ. Comparison of cardiac MRI tissue tracking and myocardial tagging for assessment of regional ventricular strain. Int $\mathrm{J}$ Cardiovasc Imaging 2012;28(8):2009-2018.

31. Hor KN, Gottliebson WM, Carson C, et al. Comparison of magnetic resonance feature tracking for strain calculation with harmonic phase imaging analysis. JACC Cardiovasc Imaging 2010;3(2):144-151.

32. Bayes-Genis A, Vazquez R, Puig T, et al. Left atrial enlargement and NT-proBNP as predictors of sudden cardiac death in patients with heart failure. Eur $\mathrm{J}$ Heart Fail 2007;9(8):802-807.

33. Cabin HS, Clubb KS, Hall C, Perlmutter RA, Feinstein AR. Risk for systemic embolization of atrial fibrillation without mitral stenosis. Am J Cardiol 1990;65(16):11121116. 